\title{
USO DO PÓ DA CASCA DE COCO NA FORMULAÇÃO DE SUBSTRATOS PARA FORMAÇÃO DE MUDAS ENXERTADAS DE CAJUEIRO ANÃO PRECOCE ${ }^{1}$
}

\author{
DIVA CORREIA ${ }^{2}$, MORSYLEIDE DE FREITAS ROSA ${ }^{3}$, ELIS REGINA DE VASCONCELOS NORÕES ${ }^{4}$, \\ FÁTIMA BEATRIZ DE ARAUJO
}

\begin{abstract}
RESUMO - Avaliou-se o uso de pó de coco verde e maduro na formulação de substratos para formação de mudas de cajueiro anão precoce. O experimento foi conduzido na Embrapa Agroindústria Tropical, em Fortaleza (CE). Porta-enxertos dos cajueiros foram obtidos com semente CCP06 e os enxertos, de árvores adultas CCP76, ambos clones de cajueiro anão precoce. Pó das cascas de coco verde e de coco maduro mostraram-se favoráveis ao desenvolvimento das plantas, apresentando boas características como facilidade de retirada da muda do tubete e agregação das raízes ao substrato, podendo assim, substituir o uso do solo hidromórfico, na proporção de $20 \%$.
\end{abstract}

Termos para indexação: resíduo agroindustrial de coco, substrato para planta, produção de muda, Cocus nucifera L, Anacardium occidentale L.

\section{THE USE OF COIR DUST FOR PREPARATION OF SUBSTRATES FOR GRAFTED DWARF CASHEW SEEDLINGS}

\begin{abstract}
The aim of this work was to evaluate the use of mature and immature coir dust for preparation of substrates for grafted dwarf cashew seedlings. The trial was carried out at Embrapa Agroindústria Tropical, in Fortaleza city, Ceará State. Root-stocks were obtained from seeds of CCP06 and vegetative shoots were obtained from adult plants of CCP76, both dwarf cashew clones. Results revealed that coir dust either from mature or immature fruit was suitable for seedlings growth, being able to replace the low humid glei soil at $20 \%$. Both substrates showed great facility to remove the seedlings from liner pots as well as good root aggregation to substrates.
\end{abstract}

Index terms: coconut agroindustry waste, substrates for plants, seedling production, Cocus nucifera L., Anacardium occidentale L.

O cultivo de plantas utilizando substratos é uma técnica amplamente empregada na maioria dos países com horticultura avançada. $\mathrm{O}$ termo substrato aplica-se a todo material sólido, natural, sintético, residual, mineral ou orgânico, distinto do solo, que colocado em um recipiente em forma pura ou em mistura permite o desenvolvimento do sistema radicular, desempenhando, portanto, um papel de suporte para a planta (Abad \& Noguera, 1998). Como características desejáveis, os substratos devem apresentar baixo custo, disponibilidade nas proximidades das regiões de consumo, suficiente teor de nutrientes, boa capacidade de troca de cátions, relativa esterilidade biológica, e permitir a aeração e a retenção de umidade (Konduru et al., 1999; Booman, 2000; Gonçalves et al., 2000), além de ser capaz de favorecer a atividade fisiológica das raízes (Gonçalves et al., 2000).

De um modo geral, resíduos agroindustriais vêm sendo progressivamente utilizados como uma alternativa para minimizar o impacto ambiental provocado por tais resíduos sólidos. Da indústria de processamento de coco verde ou maduro origina-se uma quantidade significativa de resíduos dos quais as cascas de coco maduro são geralmente utilizadas como combustível de caldeiras ou processadas para o beneficiamento de fibras longas, curtas ou pó (Rosa et al., 2001).

Diferentemente do coco maduro, as fibras do coco verde não são aproveitadas pelas indústrias de fibra, sendo as cascas descartadas em lixões ou aterros sanitários. Neste estudo, objetivou-se avaliar o uso de pó de coco verde e maduro na formulação de substratos para formação de mudas enxertadas de cajueiro anão precoce (Anacardium occidentale L.).

O experimento foi realizado no viveiro de plantas da Embrapa Agroindústria Tropical, Fortaleza (CE), em 2002, instalado em delineamento experimental em blocos casualizados com quatro repetições e ca de arroz carbonizada + folha triturada de carnaubeira (Copernicia prunifera (Miller) H. E. Moore) decomposta + solo hidromórfico (5:3:2, $\mathrm{v} / \mathrm{v}) ; \mathrm{S}_{2}$ - casca de arroz carbonizada + folha triturada de carnaubeira decomposta + pó da casca (mesocarpo) de coco verde (Cocus nucifera L.) $(5: 3: 2, \mathrm{v} / \mathrm{v}) ; \mathrm{S}_{3}$ - casca de arroz carbonizada + folha triturada de carnaubeira decomposta + pó da casca (mesocarpo) de coco (Cocus nucifera L.) maduro (5:3:2, v/v). O pó das cascas de cocos verde e madunove plantas por parcela, sendo composto por três substratos: $\mathrm{S}_{1}$ - cas-

ro foram previamente embebidos em água durante 30 minutos. Posteriormente, descartou-se a solução residual e este procedimento, repetido quatro vezes. A secagem dos mesmos foi realizada à sombra.

Porta-enxertos foram estabelecidos com sementes CCP06 (Clone de Cajueiro de Pacajus 'Anão Precoce') em tubetes com capacidade de $288 \mathrm{~cm}^{3}$, a céu aberto. Aos 40 dias após a semeadura, retiraram-se duas plantas de cada parcela para avaliação de porta-enxertos e procedeu-se a enxertia por garfagem em fenda lateral no restante do material, utilizando-se como enxerto, ramos vegetativos de plantas adultas CCP76 (Clone de Cajueiro de Pacajus 'Anão Precoce'). Após a enxertia, as plantas permaneceram em telado com $50 \%$ de luminosidade durante 25 dias. Em seguida, foram transferidas à pleno sol até completar 120 dias da semeadura. Aos 30 dias de cultivo iniciaram-se as adubações foliares com o produto comercial Biofert $\left(5 \mathrm{~mL} \cdot \mathrm{L}^{-1} \mathrm{~N}(15 \%) ; \mathrm{P}_{2} \mathrm{O}_{5}\right.$ sol. CNA $\mathrm{H}_{2} \mathrm{O}(15 \%)$; $\mathrm{K}_{2} \mathrm{O}(20 \%) ; \mathrm{Ca}(1,5 \%) ; \mathrm{Mg}(0,05 \%) ; \mathrm{Zn}(0,2 \%) ; \mathrm{B}(0,05 \%) ; \mathrm{Fe}(0,1 \%) ; \mathrm{Mn}$ $(0,02 \%) ; \mathrm{Cu}(0,05 \%) ; \mathrm{Mo}(0,01 \%) ; \mathrm{S}(3 \%)]$, sendo repetidas a cada 10 dias durante o período experimental. A partir dos 90 dias, adicionou-se $15 \mathrm{~mL} /$ tubete de solução de Hoagland (Hoagland \& Arnon, 1950), a cada 10 dias.

As características avaliadas para porta-enxerto e muda enxertada aos 40 e 120 dias após a semeadura, respectivamente, foram: altura da parte aérea, diâmetro do caule no ponto da enxertia, número de folhas, pesos da matéria seca da parte aérea e das raízes, facilidade de retirada da planta do tubete e agregação do substrato às raízes. Para facilidade de retirada da planta do tubete e agregação do substrato às raízes atribuíram-se notas: 01 (difícil ou ruim), 02 (fácil ou boa) e 03 (muito fácil ou ótima), respectivamente. Para análise estatística, esses valores foram transformados em $\sqrt{x}$. Aos 25 dias após a realização da enxertia, avaliou-se a porcentagem de pegamento dos enxertos. Os dados foram submetidos à análise de variância e as médias dos tratamentos comparadas entre si pelo teste de Tukey, a 5\% de probabilidade. As características químicas e os valores da condutividade elétrica dos substratos, realizadas no início do experimento, encontram-se na Tabela 1.

O crescimento das mudas enxertadas de cajueiro anão precoce, aos 120 dias de cultivo, parece não ter sido influenciado pelas características químicas dos substratos (Tabela 1 ). Na Tabela 2 pode-se obser-

\footnotetext{
${ }^{1}$ (Trabalho 002/2003). Recebido: 06/01/2003. Aceito para publicação: 11/09/2003.

${ }^{2}$ Pesquisador Embrapa Agroindústria Tropical, Doutorando USP/Esalq, Caixa Postal 3761, CEP 60511-110, Fortaleza (CE). e-mail diva@cnpat.embrapa.br

${ }^{3}$ Pesquisador Embrapa Agroindústria Tropical, Caixa Postal 3761, CEP 60511-110, Fortaleza (CE).

${ }^{4}$ Graduanda Engenharia Química da Universidade Federal do Ceará/Estagiária Embrapa Agroindústria Tropical.
} 
TABELA 1 - Características químicas e a condutividade elétrica dos diferentes substratos utilizados para crescimento de porta-enxertos (CCP06) e de mudas enxertadas (CCP 76) de cajueiro anão precoce em tubetes. Fortaleza (CE), 2002.

\begin{tabular}{|c|c|c|c|c|}
\hline \multicolumn{2}{|c|}{ Determinação } & $\mathrm{S}_{1}$ & $\mathrm{~S}_{2}$ & $\mathrm{~S}_{3}$ \\
\hline P (resina) & $\mathrm{mg} / \mathrm{dm}^{3}$ & 266,30 & 277,90 & 174,85 \\
\hline $\mathrm{K}$ & $\mathrm{mmol}_{\mathrm{c}} / \mathrm{dm}^{3}$ & 32,00 & 30,50 & 20,50 \\
\hline $\mathrm{Ca}$ & $\mathrm{mmol}_{\mathrm{c}} / \mathrm{dm}^{3}$ & 27,02 & 24,66 & 26,34 \\
\hline $\mathrm{Mg}$ & $\mathrm{mmol}_{\mathrm{c}} / \mathrm{dm}^{3}$ & 44,45 & 18,29 & 17,44 \\
\hline $\mathrm{Na}$ & $\mathrm{mmol}_{\mathrm{c}} / \mathrm{dm}^{3}$ & 0,30 & 85,00 & 65,00 \\
\hline $\mathrm{Cu}$ & $\mathrm{mg} / \mathrm{dm}^{3}$ & 0,08 & 0,12 & 0,47 \\
\hline $\mathrm{Fe}$ & $\mathrm{mg} / \mathrm{dm}^{3}$ & 36,18 & 41,72 & 72,38 \\
\hline $\mathrm{Mn}$ & $\mathrm{mg} / \mathrm{dm}^{3}$ & 41,40 & 41,32 & 35,52 \\
\hline $\mathrm{Zn}$ & $\mathrm{mg} / \mathrm{dm}^{3}$ & 5,16 & 5,54 & 4,94 \\
\hline Matéria Orgânica & $\mathrm{g} / \mathrm{dm}^{3}$ & 205,80 & 267,64 & 174,24 \\
\hline $\mathrm{pH}\left(\mathrm{CaCl}_{2}\right)$ & & 4,95 & 4,90 & 4,85 \\
\hline $\mathrm{H}+\mathrm{Al}$ & $\mathrm{mmol}_{\mathrm{c}} / \mathrm{dm}^{3}$ & 0,00 & 47,00 & 50,00 \\
\hline Soma de Bases & $\mathrm{mmol}_{\mathrm{c}} / \mathrm{dm}^{3}$ & 103,76 & 158,45 & 129,28 \\
\hline CTC & $\mathrm{mmol}_{\mathrm{c}} / \mathrm{dm}^{3}$ & 103,76 & 205,45 & 179,28 \\
\hline Saturaç & $\%$ & 74,74 & 77,12 & 72,11 \\
\hline Condutividade Elétrica & $\mathrm{dS} / \mathrm{m}$ & 1,40 & 0,95 & 1,10 \\
\hline
\end{tabular}

Análises realizadas no Laboratório de Solo e Água da Embrapa Agroindústria Tropical.

$\mathrm{S}_{1}=$ casca de arroz carbonizada + folha de carnaubeira triturada + solo hidromórfico;

$\mathrm{S}_{2}=$ casca de arroz carbonizada + folha da carnaubeira triturada + pó da casca do coco verde;

$\mathrm{S}_{3}=$ casca de arroz carbonizada + folha de carnaubeira triturada + pó da casca do coco maduro.

var diferenças estatísticas significativas somente entre as alturas das partes aéreas em porta-enxertos cultivados nos substratos $S_{1}(22,8 \mathrm{~cm})$ e $\mathrm{S}_{3}(20,9 \mathrm{~cm})$. O mesmo pode ser observado na Tabela 3 para a variável facilidade de retirada da muda do tubete entre os substratos $\mathrm{S}_{2}(2,10)$ e $\mathrm{S}_{1}$ $(1,95)$. Todavia, valores indicados nas Tabelas 2 e 3 para altura da parte
TABELA 2 - Valores médios de altura da parte aérea (h), diâmetro de caule no ponto de enxertia (dc), número de folhas, peso seco da parte aérea (pspa), peso seco da raiz (psr), facilidade de retirada da muda do tubete (fr) e agregação do substrato às raízes (asr) para porta-enxertos de cajueiro anão precoce (CCP06), cultivados em diferentes substratos, aos 40 dias após a semeadura. Fortaleza (CE), 2002.

\begin{tabular}{cccccccc}
\hline Substratos & $\begin{array}{c}\mathrm{h} \\
(\mathrm{cm})\end{array}$ & $\begin{array}{c}\mathrm{dc} \\
(\mathrm{cm})\end{array}$ & $\begin{array}{c}\text { folhas } \\
\left(\mathrm{n}^{\mathrm{o}}\right)\end{array}$ & $\begin{array}{c}\text { pspa } \\
(\mathrm{g})\end{array}$ & $\begin{array}{c}\mathrm{psr} \\
(\mathrm{g})\end{array}$ & $\mathrm{fr}^{(1)}$ & $\operatorname{asr}^{(1)}$ \\
\hline $\mathrm{S}_{1}$ & $22,8 \mathrm{a}$ & $0,47 \mathrm{a}$ & $8,64 \mathrm{a}$ & $2,25 \mathrm{a}$ & $0,42 \mathrm{a}$ & $1,99 \mathrm{a}$ & $1,79 \mathrm{a}$ \\
$\mathrm{S}_{2}$ & $22,4 \mathrm{ab}$ & $0,48 \mathrm{a}$ & $8,97 \mathrm{a}$ & $2,12 \mathrm{a}$ & $0,42 \mathrm{a}$ & $2,03 \mathrm{a}$ & $1,96 \mathrm{a}$ \\
$\mathrm{S}_{3}$ & $20,9 \mathrm{~b}$ & $0,47 \mathrm{a}$ & $8,72 \mathrm{a}$ & $1,95 \mathrm{a}$ & $0,44 \mathrm{a}$ & $1,96 \mathrm{a}$ & $1,96 \mathrm{a}$ \\
\hline
\end{tabular}

$\mathrm{S}_{1}=$ casca de arroz carbonizada + folha de carnaubeira triturada + solo hidromórfico;

$\mathrm{S}_{2}=$ casca de arroz carbonizada + folha da carnaubeira triturada + pó da casca do coco verde; $\mathrm{S}_{3}=$ casca de arroz carbonizada + folha de carnaubeira triturada + pó da casca do coco maduro.

(1) Análise com valores transformados em $\sqrt{x}$.

Médias seguidas da mesma letra, nas colunas, não diferem entre si, significativamente, ao nível de $5 \%$ de probabilidade, pelo teste de Tukey.

aérea, diâmetro do caule e número de folhas tanto para porta-enxerto quanto para muda enxertada, independente do substrato, estão de acordo com os valores recomendados por Cavalcanti Júnior \& Chaves (2001) para mudas de cajueiro anão precoce crescidas em tubetes no substrato $\mathrm{S}_{1}$, o qual tem sido recomendado para uso em sistemas comerciais de produção de mudas enxertadas. Os substratos não influenciaram no pegamento dos enxertos (Tabela 3 ).

Tais resultados sugerem que o pó da casca do coco maduro ou verde, na proporção de $20 \%$, pode ser um dos componentes na mistura do substrato recomendado na produção de mudas enxertadas de cajueiro anão precoce em tubetes, substituindo o solo hidromórfico. Adicionalmente, proporciona uma alternativa de uso adequado para os resíduos agroindustriais de coco maduro e verde.

TABELA 3 - Valores médios de altura (h), diâmetro de caule no ponto de enxertia (dc), número de folhas, peso seco da parte aérea (pspa), peso seco da raiz (psr), facilidade de retirada da muda do tubete (fr), agregação do substrato às raízes (asr) aos 120 dias após a semeadura e de pegamento dos enxertos (pe) aos 25 dias após a enxertia, para mudas enxertadas de cajueiro anão precoce (CCP76), cultivadas em diferentes substratos. Fortaleza (CE), 2002.

\begin{tabular}{cccccccccc}
\hline Substrato & $\mathrm{h}(\mathrm{cm})$ & $\mathrm{dc}(\mathrm{cm})$ & folhas $\left(\mathrm{n}^{\circ}\right)$ & $\mathrm{pspa}(\mathrm{g})$ & $\mathrm{psr}(\mathrm{g})$ & $\mathrm{pe}$ & $(\%)$ & $\mathrm{fr}^{(1)}$ & $\mathrm{asr}^{(1)}$ \\
\hline $\mathrm{S}_{1}$ & $18,5 \mathrm{a}$ & $0,66 \mathrm{a}$ & $7,27 \mathrm{a}$ & $2,37 \mathrm{a}$ & $0,65 \mathrm{a}$ & $82,14 \mathrm{a}$ & $1,95 \mathrm{~b}$ & $1,96 \mathrm{a}$ \\
$\mathrm{S}_{2}$ & $19,1 \mathrm{a}$ & $0,64 \mathrm{a}$ & $7,53 \mathrm{a}$ & $2,50 \mathrm{a}$ & $0,52 \mathrm{a}$ & $81,71 \mathrm{a}$ & $2,10 \mathrm{a}$ & $1,93 \mathrm{a}$ \\
$\mathrm{S}_{3}$ & $20,6 \mathrm{a}$ & $0,68 \mathrm{a}$ & $8,37 \mathrm{a}$ & $2,79 \mathrm{a}$ & $0,63 \mathrm{a}$ & $82,33 \mathrm{a}$ & $2,04 \mathrm{ab}$ & $1,98 \mathrm{a}$ \\
\hline
\end{tabular}

$\mathrm{S}_{1}=$ casca de arroz carbonizada + folha de carnaubeira triturada + solo hidromórfico;

$\mathrm{S}_{2}=$ casca de arroz carbonizada + folha da carnaubeira triturada + pó da casca do coco verde; $\mathrm{S}_{3}=$ casca de arroz carbonizada + folha de carnaubeira triturada + pó da casca do coco maduro.

(1) Análise com valores transformados em $\sqrt{x}$

Médias seguidas da mesma letra, nas colunas, não diferem entre si, significativamente, ao nível de $5 \%$ de probabilidade, pelo teste de Tukey.

\section{REFERÊNCIAS BIBLIOGRÁFICAS}

ABAD, M.; NOGUERA, P. Substratos para el cultivo sin suelo y fertirrigación. In: CADAHIA, C. (Ed.) Fertirrigación: cultivos hortícolas y ornamentales. Madrid: Mundi-Prensa, 1998. p.287-342.

BOOMAN, J. L. E. Evolução dos substratos usados em horticultura ornamental na Califórnia. In: KAMPF, A. N.; FERMINO, M. H. (Ed.) Substratos para plantas: a base da produção vegetal em recipientes. Porto Alegre: Gênesis, 2000. p.43-65.

CAVALCANTI JÚNIOR, A. T.; CHAVES, J. C. M. Produção de mudas de cajueiro. Fortaleza: Embrapa Agroindústria Tropical, 2001. 43p. (Documentos, 42).

EVANS, M. R.; STAMPS, R. H. Growth of bedding plants in Sphagnum peat and coir dust-based substrates. Journal of Environmental Horticulture, Madison, v.14, p.187-190, 1996.
GONÇALVES, J. L. M.; SANTARELI, E. G.; MORAES NETO, S. P.; MANARA, M. P. Produção de mudas de espécies nativas: substrato, nutrição, sombreamento e fertilização. In: GONÇALVES, J. L. M.; BENEDETTI, V. (Ed.). Nutrição e fertilização florestal. Piracicaba: IPEF, 2000. p.309-350.

HOAGLAND, D. R.; ARNON, D. T. The water culture method for growth plants without soil. Berkely: California Agriculture Experiment Station, 1950. 32p. (University of California. Circular 347).

KONDURU, S.; EVANS, M. R; STAMPS, R. H. Coconut husk and processing effects on chemical and physical properties of coconut coir dust. HortScience, Alexandria, v.34, p.88-90, 1999.

ROSA, M. F.; SANTOS, J. S. S.; MONTENEGRO, A. A. T.; ABREU, F. A. P.; ARAUUJO, F. B. S., NORÕES, E. R. Caracterização do pó da casca de coco verde usado como substrato agrícola. Fortaleza: Embrapa Agroindústria Tropical, 2001.6p. (Comunicado Técnico, 5). 